\title{
Preface: molecular, cellular, and tissue mechanobiology
}

\author{
Xi-Qiao Feng ${ }^{1}$ - Peter Vee Sin Lee ${ }^{2}$ Chwee Teck Lim ${ }^{3}$
}

Published online: 6 April 2017

(C) The Chinese Society of Theoretical and Applied Mechanics; Institute of Mechanics, Chinese Academy of Sciences and Springer-Verlag Berlin Heidelberg 2017

Mechanics plays a crucial role in a diversity of biological processes at different length (from molecules, cells, tissues, organs to organisms) and time scales. As a rapidly growing field across the interfaces of mechanics, biology, and medical engineering, mechanobiology aims to identify the mechanical and biological responses of cells and tissues of mechanical factors (e.g., stress, strain, and substrate stiffness) and their underlying mechanisms at different scales; to correlate the physiological and pathological growth, adaption, remodeling, and degradation of tissues and organs with their mechanical forces, properties, and environments; and to provide mechanics-based approaches and techniques for diagnosis and therapy. To this end, mechanobiology integrates the experimental and theoretical methods of biology and mechanics to investigate the physiological and pathological responses of biological molecules, cells, and tissues to mechanical factors at different levels. Mechanobiology not only provides insights into a wealth of biological processes at the molecular, cellular, and tissue levels but also cues to developing novel therapeutic strategies for diseases such as cancer.

In particular, cell mechanobiology (or cytomechanobiology) has attracted much theoretical and experimental efforts

$\triangle$ Xi-Qiao Feng

fengxq@tsinghua.edu.cn

1 Institute of Biomechanics and Medical Engineering, Department of Engineering Mechanics, Tsinghua University, Beijing 100084, China

2 Department of Mechanical Engineering, University of Melbourne, Melbourne, VIC 3010, Australia

3 Department of Mechanical Engineering and Mechanobiology Institute, National University of Singapore, Singapore, Singapore devoted toward clarifying how cells sense their environment and adequately respond in terms of shape, migration, proliferation, differentiation, and survival. Cells can not only sense and respond to mechanical stresses but also regulate the mechanical properties of the surrounding extracellular matrix $(\mathrm{ECM})$. For example, the integration of physical and biochemical factors within the microenvironment can modulate the differentiation of stem cells; naive mesenchymal stem cells can specify lineage and commit to specific phenotypes depending on the elastic modulus of ECM. Compressive stress can direct cancer cells toward an invasive phenotype and enable coordinated migration of these malignant cells.

This thematic issue of Acta Mechanica Sinica contains six invited articles in the field of mechanobiology. The editors hope that it will bring new interests and inspirations to the research of molecular, cellular, and tissue mechanobiology.

Cells use a dynamics actin network to drive their motility, polarization, and morphogenesis. Considering the molecularlevel processes of actin polymerization, branching, capping, and depolymerization, Gong et al. [1] developed a computational framework to investigate the stochastic and cooperative behaviors of growing actin networks in overcoming obstacles. They discovered that the characteristic sizes of obstacles change the protrusion power per unit length but do not influence the orientation distribution of actin filaments in the growing network.

The regulated transport of molecules of different sizes across cell membranes usually involves the formation of nanopores. For example, Bu et al. [2] performed all-atom molecular dynamics and bias-exchange metadynamics simulations to reveal the molecular mechanisms of water pore formation. They showed that a water pore of a few nanometers in diameter can form on a cell membrane subjected to an electric field through which water molecules can pass through 
the membrane. They provided some atomic details of how an electric field influences the movement of water molecules and lipid head groups during water pore formation.

The force regulation and coordination of molecular motors are crucial for the elongation and contraction of skeletal muscles. Wei et al. [3] studied the role of adenosine triphosphate (ATP) in regulating the coordination among multiple molecular motors. A mechanics model of sarcomeres was used to show how the ATP concentration affects the motor force regulation during skeletal muscle contraction. They found that there could be negative cross-bridges resisting contraction within the sarcomere, and a higher ATP concentration might decrease the resistance force from negative cross-bridges by promoting their timely detachment.

Epithelial morphogenesis not only integrates many physiological activities such as cell proliferation and apoptosis, but also entails collective cell mechanics. So that Lin et al. [4] presented a dynamic cellular vertex model to study the morphomechanics of a growing epithelial monolayer. The feedback between cell proliferation and mechanical stresses was introduced into the model to elucidate how the feedback at the cellular level tailors the macroscopic features at the tissue level. They highlighted the critical role of mechanical factors in the growth and morphogenesis of epithelial tissues.

To date, it remains unclear how dental neurons sense hot and cold stimuli. To find out about this, Lin et al. [5] investigated the relationship between temperature distribution, tooth structure deformation, dentinal fluid flow, and the time course of dental neuron response under thermal stimulation. They used biophysical models to demonstrate that both hydrodynamic theory and neuron theory contribute to dental thermal pain sensations. Their results support a central role of mechanosensitive ion channels and the associated hydrodynamic hypothesis.

Osteoporosis is accompanied by the reduction of bone quantity and is a major cause of fractures in the spines of older persons. By applying the progressive loading technique, $\mathrm{Li}$ et al. [6] experimentally studied how osteoporosis induced by estrogen depletion influences the evolution of post-yield microdamage accumulation and plastic deformation in vertebral bodies. They found that osteoporosis could increase the vertebral fracture risk probably through microdamage accumulation and plastic deforming degradation.

Acknowledgements Supports from the National Natural Science Foundation of China (Grants 11432008 and 11620101001) are acknowledged. We wish to thank all authors of this thematic issue for their contributions.

\section{References}

1. Gong, B., Lin, J., Qian, J.: Growing actin networks regulated by obstacle size and shape. Acta Mech. Sin. (2017). doi:10.1007/ s10409-016-0628-5

2. Bu, B., Li, D., Diao, J., et al.: Mechanics of water pore formation in lipid membrane under electric field. Acta Mech. Sin. (2017). doi:10. 1007/s10409-017-0635-1

3. Wei, J., Dong, C., Chen, B.: Effects of adenosine triphosphate concentration on motor force regulation during skeletal muscle contraction. Acta Mech. Sin. (2017). doi:10.1007/s10409-017-0637-z

4. Lin, S.Z., Li, B., Feng, X.Q.: A dynamic cellular vertex model of growing epithelial tissues. Acta Mech. Sin. (2017). doi:10.1007/ s10409-017-0654-y

5. Lin, M., Liu, F., Liu, S., et al.: The race to the nociceptor: mechanical versus temperature effects in thermal pain of dental neurons. Acta Mech. Sin. (2017). doi:10.1007/s10409-017-0634-2

6. Li, S., Niu, G., Dong, N.X., et al.: Osteoporosis affects both post-yield microdamage accumulation and plasticity degradation in ovariectomized rat vertebra. Acta Mech. Sin. (2017). doi:10.1007/ s10409-017-0643-1

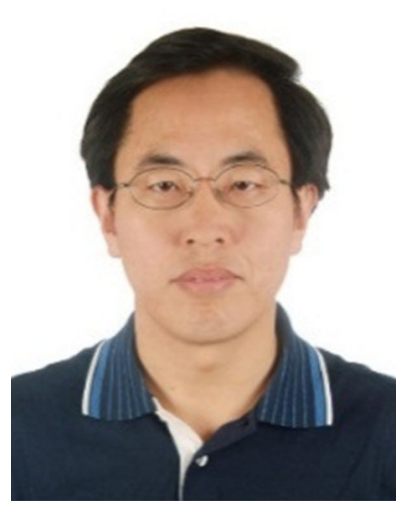

Xi-Qiao Feng is a Chang Jiang Chair Professor and the head of Department of Engineering Mechanics, Tsinghua University. $\mathrm{He}$ received bachelor, master, and doctoral degrees of solid mechanics in 1990, 1991, and 1995 at Tsinghua University, respectively. From 1997 to 1999 , he worked as an Alexander von Humboldt research fellow in the Technical University of Darmstadt and Delft University of Technology. He rejoined Tsinghua University as an associate professor in 1999 and was promoted to a professor in 2001. During 2010-2014, he served as the secretary-general of the Chinese Society of Theoretical and Applied Mechanics. Since 2006, he has been the director of the Institute of Biomechanics and Medical Engineering (IBME) at Tsinghua University. He also serves as an associate editor or editorial board member of about ten international journals. He has authored about 260 papers. His research interests include damage and fracture mechanics; mechanics of cells, biological materials, and tissues. Selected Feng's honors include the Award of Science and Technology for Young Scientists of China (2007), Distinguished Young Scholars Award of NSFC (2005), Young Scientist Award of Fok Ying Tong Education Foundation (2004), Award of Distinguished Doctoral Theses of China (1999), etc. 


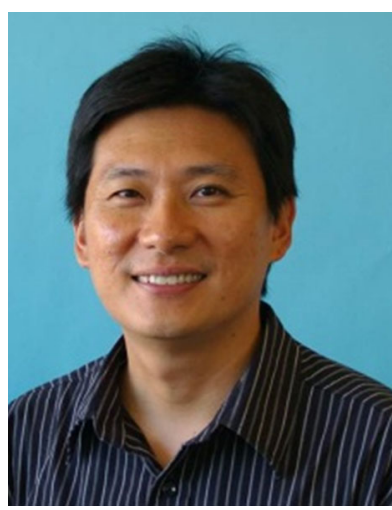

Peter Vee Sin Lee obtained his B.E. in Mechanical Engineering (1991) and Ph.D. in Bioengineering (1996) from the University of Strathclyde, UK, and continued his post-doc in the same university during 1996-1998. He was a research fellow in the Biomaterials Group at Institute of Materials Research and Engineering, Singapore from 1998-2001. In 2001, he joined the Defence Medical and Environmental Research Institute, DSO National Laboratories, Singapore, as the head of the Bioengineering Laboratory. He joined the University of Melbourne as a Senior Lecturer in 2008, and was promoted to an associate professor in 2011 and a professor in 2015. Currently, he leads the Cell and Tissue Biomechanics Laboratory where his research aims to better understand the behavior of biological cells and tissues under mechanical forces. He has authored more than 140 publications in journals, conference proceedings and books. $\mathrm{He}$ is a regular reviewer for journals and grant bodies and currently the Associate Editor for Journal of Orthopaedic Surgery and Research, and Frontiers in Pharmacology (Translational Pharmacology).

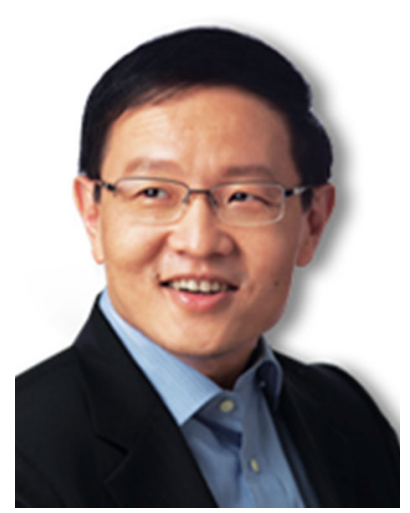

Chwee Teck Lim is NUSS Chair Professor in the Department of Biomedical Engineering and a Principal Investigator at the Mechanobiology Institute at the National University of Singapore. His research interests include the mechanobiology of human diseases and the development of microfluidic technologies for disease detection, diagnosis and therapy. He has authored more than 310 peerreviewed papers and delivered more than 300 invited talks. He is an elected Fellow of the American Institute for Medical and Biological Engineering, International Academy of Medical and Biological Engineering as well as an elected member of the World Council of Biomechanics. He currently sits on the editorial boards of more than 14 international journals. He has co-founded five startups which are commercializing technologies developed in his lab. He and his team have garnered more than 60 research awards and honors including the International Precision Medicine Conference Prize (2017), Asian Scientist 100 (2016), Vladimir K. Zworykin Award (2015), University's Outstanding Researcher Award (2014), the Credit Suisse Technopreneur of the Year Award and Wall Street Journal Asian Innovation Award (Gold) (2012), and the President's Technology Award (2011). 